\title{
Le gène des amyotrophies spinales est localisé sur le bras long du chromosome 5
}

Les amyotrophies spinales sont un groupe de maladies héréditaires caractérisées par une dégénérescence des neurones moteurs de la corne antérieure de la moelle épinière. Il en résulte une dénervation progressive du muscle strié responsable d'un déficit moteur avec atrophie musculaire (d'où son nom amyotrophie spinale).

L'hétérogénéité clinique de ces affections est connue de longue date. Différentes classifications ont été proposées prenant en compte la distribution du déficit moteur, l'âge de début des symptômes et l'évolutivité de la maladie [1]. La maladie de WerdnigHoffmann aiguë ou type I est la forme la plus sévère et la plus fréquente. Elle aboutit au décès par atteinte des muscles respiratoires dans les deux premières années de vie. La forme dite intermédiaire ou type II se caractérise, comme la précédente, par un début des signes dans la première année de vie mais son évolution est prolongée et s'accompagne d'un handicap moteur et respiratoire extrêmement sévère. Enfin, la maladie de Kugelberg Welander ou type III se distingue des précédentes par une symptomatologie d'apparition plus tardive, après l'âge de la marche et un bon pronostic vital.

Ces affections ont un mode de transmission récessif autosomique et elles arrivent par leur fréquence au second rang des maladies récessives après la mucoviscidose. La ou les anomalie(s) biochimique(s) responsable(s) de ces affections reste(nt) inconnue(s). Les variants cliniques de la maladie ont été considérés comme la conséquence soit de mutations de gènes différents, soit de différentes mutations au même locus.
Par analyse de liaison génétique, il est possible, à l'aide de marqueurs génétiques polymorphes, de localiser sur le génome le locus morbide d'une maladie héréditaire. Cette analyse consiste à étudier, dans des familles multiples, la ségrégation conjointe d'un marqueur génétique et du locus morbide. Le taux de recombinaison mesure le pourcentage de gamètes recombinés parmi l'ensemble des gamètes transmis par les parents. En cas d'indépendance, on attend autant de gamètes recombinés que de gamètes parentaux. Dans le cas contraire, les gamètes se transmettent dans leur configuration parentale plutôt que recombinés. On dit alors qu'il y a liaison génétique ou linkage entre le locus polymorphe et la maladie. La méthode dite des "lod scores "* proposée par Morton en 1955 [2] permet de tester à la fois la liaison génétique entre deux loci et d'estimer le taux de recombinaison entre ces loci. Pour mener à bien notre étude, vingt-quatre familles d'origine géographique différente (France, Belgique, Italie, Espagne, Portugal, Maroc, Tunisie) comportant au moins deux sujets atteints ont été sélectionnées parmi plus de deux cents familles recensées et prélevées. Seize appartiennent au type II et huit au type III. Près de 180 marqueurs génétiques ont été choisis selon deux critères : la fréquence des hétérozygotes pour les RFLPs révélés par la sonde (supérieure à $30 \%$ ) et la distribution de ceux-ci sur chacun des 22 autosomes à une distance génétique d'au moins 20 centimorgans** entre chacun d'eaux. Après avoir exclu $60 \%$ du génome à l'aide de 132 marqueurs, nous avons établi une liaison génétique entre le locus morbide et le segment anonyme D5S39 localisé sur le bras long du chromosome 5 (5q12-q14). Le programme informatique LINKAGE [3] facilite grandement le calcul des lod scores à des taux de recombinaison variable. Ici, un lod score maximum de +6,6 a été observé à un taux de recombinaison de 0,00 (intervalle de confiance : $0,00-0,05)$. L'analyse indépendante des familles de type II et III donne des valeurs de lod score respectivement de $+4,06$ et $+2,54$ à un taux de recombinaison $=0,00$. Ainsi nos résultats [4] et ceux obtenus indépendamment par le groupe de Conrad Gilliam [5] montrent que le gène responsable des formes chroniques des amyotrophies spinales est localisé sur le bras long du chromosome 5 .

Ces deux études concordantes permettent :

- d'envisager à court terme un diagnostic antenatal de ces affections redoutables, tant attendu par les familles ;

- d'entreprendre la cartographie génétique et physique de cette région du génome pour l'isolement d'un gène impliqué dans la survie des motoneurones ;

- de tester des modèles animaux dans les régions homologues à celles du chromosome 5 .

\footnotetext{
* Le lod score est le logarithme décimal du rapport entre la probabilité que l'hypothèse d'une liaison génétique soit juste et celle qu'elle soit fausse. Un lod score de 6 signifie que les chances de liaison contre celles de non liaison sont dans le rapport 106/1. ** 1 centimorgan : distance génétique entre deux marqueurs pour lesquels existe $1 \%$ de recombinaison à la méiose.
} 
Pourquoi ces études ont-elles été initiées sur les formes chroniques plutôt que les formes aiguës plus fréquentes et plus homogènes cliniquement ? C'est que le pronostic de la forme aiguë est redoutable. De ce fait la taille des familles est petite rendant l'analyse génétique difficile. Désormais, il est possible de tester l'hypothèse, probable, d'un même locus pour les trois formes.

Ainsi la génétique à rebours ou génétique inverse, stratégie maintenant classique, montre une nouvelle fois sa force. A cet égard, il est bon de souligner l'importance d'une collaboration étroite entre médecins et chercheurs et la lourdeur actuelle de cette approche qui pourrait se trouver allégée par l'automatisation de certaines étapes.

J.M. A.M. J.F.

1. Emery AEH. The nosology of the spinal muscular atrophies. J Med Genet 1971; 8: 481-95

2. Morton NE. Sequential tests for the detection of linkage. Am J Hum Genet 1955 ; 7: 278-318.

3. Lathrop GM, Lalouel JM, Julier C et al. Strategies for multilocus linkage analysis in humans. Proc Nall Acad Sci USA 1984 ; 81: 3442-6.

4. Melki J, Abdelhak S, Sheth P et al. Gene for chronic proximal spinal muscular atrophies maps to chromosome 5q. Nature $1990 ; 344: 767-8$

5. Brzustowicz LM, Lehner T, Castilla LH et al. Genetic mapping of chronic childhoodonset spinal muscular atrophy to chromosome 5q11.2-13.3. Nature $1990 ; 344$ : 540-1.

$m / s n^{\circ} 5$ vol. 6 , mai 90
Une souris déficiente en phénylalanine hydroxylase (PAH). Le déficit en PAH à la base de la phénylcétonur ie est une des maladies génétiques les plus importantes et la seule qui fasse l'objet d'un diagnostic néonatal systématique. Il n'existait pas, jusqu'à présent, de modèle animal correct. Une équipe américaine (Madison, WS, USA et Manhattan, KS, USA) a obtenu par mutagenèse à l'éthylnitrosouréc quatre lignées de souris déficientes en PAH dont une a été étudiée en détail [1]. I ce test de criblage est une épreuve de charge en phénylalanine, qui révèle la difficulté d'élimination de l'acide aminé ainsi que le passage dans l'urine de dérivés phénylcétoniques. Le dosage de la PAH dans le foie montre un effondrement à environ $3 \%$ du taux normal. Che\% les hétérozygotes, l'activité est d'environ $30 \%$ de celle des témoins. Les auteurs ont vérifié l'absence d'inhibiteur, ainsi que d'une alcération du coenzyme, la tétrahydrobioptérine. Ces souris déficientes ont toutefois un taux de base de phénylalaninémie normal avec le régime standard du laboratoire, peut-être parce que l'activité dans le foic de souris est dix fois plus élevée que chez l'homme, de sorte que la faible activité résiduelle pourrait suffire; elles n'ont donc pas de symptomes cliniques. Des essais sont en cours avec un régime enrichi en phénylalanine (25 $\mathrm{mg}$ par ml d'cau), qui provoque chez les mutants homozygotes une hyperphénylalaninémic $(2.2 \mathrm{mM}$ au lieu de ()$, 12 \mathrm{mM}$ chez les témoins). La disponibilité d'un modèle de souris déficientes en PAH sera précieuse pour la mise sur pied d'une thérapeutique génique in vivo chez l'animal, alors que jusqu'à présent c'est seulement dans des cellules en culture que des clones d'ADNe de PAH ont été introduits.
[1. McDonald JD, et al. Proc Nall Acad Sci USA 1990); 87 : 1965-7.]

Le caractère oncogénique de la protéine Tat du virus HTL V-1 fut clairement mis en évidence in vivo, dans des modiles de souris transgéniques $\left(\mathrm{m} / \mathrm{s} n^{\circ} 10\right.$, vol. .3, p. 623). I e potentiel oncogénique de la protéine Tat du virus HIV-1 fut, quant à lui, suggéré, là encore par transgenèse, devant l'apparition de tumeurs s'apparentant aux sarcomes de Kaposi, néoplasie la plus fréquemment associćc au SIIJA. Un second phénotype, renforçant ces résultars, apparaît chez les animaux exprimant le gène tat. Plus de 60 \% des animaux âgés de 24 mois et exprimant la protéine Tat développent des tumeurs hépatiques variées (adénomes, carcinomes et hémangiomes). Comme clans le cas des lésions de type Kaposi précédemment observées, seuls les mâles présentent dès tumeurs hépatiques. De tels modèles permettent d'affirmer qu'une partic des symptômes supposés liés à une immunosuppression ou à des cofacteurs environnementaux pourraient être plus directement liés à l'expression de protéines virales, en particulier de Tat. Ceci est à rapprocher de conclusions analogues rapportées récemment dans $\mathrm{m} / \mathrm{s}$ ( $\mathrm{n}^{\circ} 9$, vol. 5 , p. 624), concernant la possibilité d'une intervention directe de la protéine Tat du virus HTLV-1 dans l'apparition de syndromes de Sjögren chez certains malades infectés, et de l'antigène $T$ des papovavirus JC. dans les leuco-encéphalopathies multifocales progressives de patients infectés ayant une résistance immunitaire altéréc.

[1. Lassam N, Fcigenbaum L, Vogel J, Jay G. Mol Biol Med 1989; 6 319-31.]
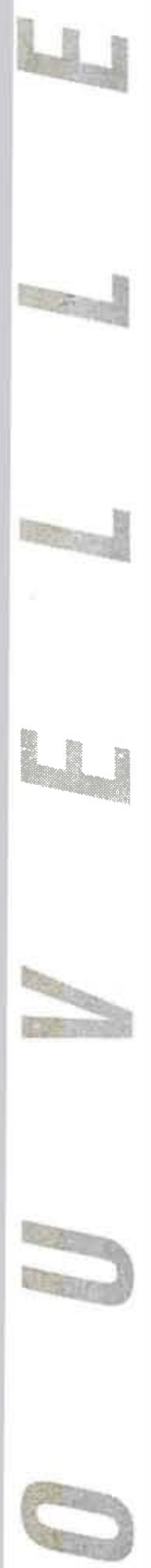